\title{
Bisimulation Theory for Switching Linear Systems
}

\author{
Giordano Pola, Arjan J. van der Schaft and Maria D. Di Benedetto
}

\begin{abstract}
A general notion of hybrid bisimulation is proposed and related to the notions of algebraic, state-space and input-output equivalences for the class of switching linear systems. An algebraic characterization of hybrid bisimulations and a procedure converging in a finite number of steps to the maximal hybrid bisimulation are derived. Bisimulation-based reduction and simulation-based abstraction are defined and characterized. Connections with observability are investigated.
\end{abstract}

\section{INTRODUCTION}

Bisimulation theories were historically introduced by the computer science community for analyzing and reducing topological complexity of concurrent processes (e.g. [4],[7]). The rise of interest in hybrid systems led to a growing reapproachment of those computer science notions in the control system community. In fact, extensions of the notion of bisimulation to dynamical systems have been explored before in [2] and in a series of papers by Pappas and co-authors (e.g. [1], [11], [12], [14]). The common denominator of those works is to associate a transition system [7] to the process under consideration that preserves reachability properties. Furthermore in [15],[16], the general definition of bisimulation for dynamical systems has been elaborated.

Inspired by classical notions of bisimulation for concurrent processes [4] and by the new notions given in [15],[16] for dynamical systems, we propose a general notion of hybrid bisimulation for the class of switching linear systems, including also continuous external variables as control laws, disturbances and outputs. The given definition seems particularly appealing since it clearly links to well-known concepts of state-space, algebraic and input/output equivalences [3] for dynamical systems: in fact bisimulationbased equivalence will be proved to be implied by algebraic equivalence and to imply state-space equivalence. Moreover connections with observability [6] are addressed, devoting particular attention to the preservation of this property under bisimulation-based reduction and simulation-based abstraction. The results are given without proofs for lack of space. A full version of this paper can be found in [13].

The paper is organized as follows. In Section II, the notion of hybrid bisimulation is proposed, characterized

This work was partially supported by European Commission under Projects HYBRIDGE IST-2001-32460, COLUMBUS IST-2001-38314 and by Ministero dell'Istruzione, dell'Università e della Ricerca under PRIN02.

G. Pola and M. D. Di Benedetto are with the Department of Electrical Engineering, Center of Excellence DEWS, University of L'Aquila, Poggio di Roio, 67040 L'Aquila, Italy pola, dibenededing. univaq. it

A. J. van der Schaft is with the Department of Applied Mathematics, University of Twente P.O. Box 217,7500 AE Enschede, The Netherlands a.j.vanderschaftemath. utwente.nl and related to algebraic, state-space and input/output equivalences. In Section III, a procedure converging to the maximal hybrid bisimulation is proposed. In Section IV, reduction via bisimulation is performed. Section $\mathrm{V}$ is devoted to simulation and abstraction. Section VI addresses connections between observability, reduction via bisimulation and abstraction via simulation. Finally, Section VII offers some concluding remarks.

\section{Bisimilar SWitching LineAR Systems}

\section{A. Preliminaries and basic definitions}

The class of switching systems we consider in this paper are defined following [6].

Definition 1: A Switching Linear System (SLS) $\mathbb{S}$ is a tuple $(\Xi, U, D, Y, \Sigma, \gamma, E, M)$ where, $\Xi=\bigcup_{q \in Q}\{q\} \times$ $X(q)$ is the hybrid state space, being $Q=\left\{q_{1}, q_{2}, \ldots, q_{N_{1}}\right\}$ the discrete state space, $N_{1} \in \mathbb{N}$, $\operatorname{dim}: Q \rightarrow \mathbb{N}, \forall q \in Q$, $X(q) \subset \mathbb{R}^{\operatorname{dim}(q)}$ is the linear continuous state space; $U$ is the linear input space; $D=V \times W$ is the hybrid disturbance space, being $V=\left\{v_{1}, v_{2}, \ldots, v_{N_{2}}\right\}$ the set of the discrete disturbances, $N_{2} \in \mathbb{N}, W$ the linear continuous disturbance space; $Y=P \times H$ is the hybrid output space, being $P=\left\{p_{1}, p_{2}, \ldots, p_{N_{3}}\right\}$ the discrete output space, $N_{3} \in \mathbb{N}$, $H$ the linear continuous output space; $\Sigma$ is a function that associates to any discrete state $q \in Q$, the linear dynamical system

$$
\begin{aligned}
\dot{x}(t) & =A(q) x(t)+B(q) u(t)+G(q) w(t), \\
h(t) & =C(q) x(t), t \geq 0 ;
\end{aligned}
$$

$\gamma: Q \rightarrow P$ associates a discrete output to each discrete state; $E \subset Q \times V \times Q$ is a collection of discrete transitions; $M$ is a function that associates to any $e=\left(q, v, q^{\prime}\right) \in E$, the reset matrix $M(e) \in \mathbb{R}^{\operatorname{dim}(q) \times \operatorname{dim}\left(q^{\prime}\right)}$.

Any $\xi=(q, x) \in \Xi$ is called hybrid state, any $d=$ $(v, w) \in D$ hybrid disturbance and any $y=(p, h) \in Y$ hybrid output of $\mathbb{S}$. Given $\mathbb{S}$, the tuple $\mathbb{D}_{\mathbb{S}}=(Q, P, V, E, \gamma)$ can be viewed as a Discrete Event System (DES) [8], having state space $Q$, output set $P$, input set $V$, transition relation $E$ and output function $\gamma$. The set $\operatorname{succ}(q)$ is composed by the successors of the discrete state $q \in Q$, i.e. $\operatorname{succ}(q)=$ $\left\{q^{\prime} \in Q \mid \exists v \in V:\left(q, v, q^{\prime}\right) \in E\right\}$. Given a set $Z \subset Z_{1} \times$ $Z_{2}$, the operator $\left.\Pi\right|_{Z_{i}}(Z)$ is the projection of the set $Z$ onto $Z_{i}, i=1,2$. We assume throughout the paper that the hybrid disturbance is not measurable and that multiple instantaneous transitions are allowed.

We recall that a hybrid time basis $\tau$ [10] is an infinite or finite sequence of time intervals $I_{j}, j \in\{0,1, \ldots, J\}$, i.e. $\tau=\left\{I_{j}\right\}_{j=0}^{J}$, satisfying the following conditions: $I_{j}=\left\{t \in \mathbb{R}_{0}^{+}: t_{j} \leq t \leq t_{j}^{\prime}\right\}$, if $j<J ; I_{J}$ may be of the form $\left\{t \in \mathbb{R}_{0}^{+}: t_{J} \leq t \leq t_{J}^{\prime}\right\}$ or of the form 
$\left\{t \in \mathbb{R}_{0}^{+}: t_{J} \leq t<\infty\right\}$ and $t_{J}^{\prime}=\infty$; for all $j, t_{j} \leq t_{j}^{\prime}$ and for $j>0, t_{j}=t_{j-1}^{\prime}$. Denote by $\mathcal{T}$ the set of all hybrid time bases. Since SLSs under consideration are time-invariant, there is no loss of generality into assuming $t_{0}=0$, for all $\tau \in \mathcal{T}$. Given $\tau \in \mathcal{T}$, denote by $[\tau]:=\bigcup_{I_{j} \in \tau} I_{j} \times\{j\}$ the set of all hybrid times $(t, j), t \in I_{j}, I_{j} \in \tau$, and define the ordering relation $<$ on $[\tau]$ such that $(t, j)<\left(t^{\prime}, j^{\prime}\right)$ if $t<t^{\prime}$ and $j \leq j^{\prime}$. Given $\tau_{1}, \tau_{2} \in \mathcal{T}$, such that $\sup \{t$ : $\left.(t, j) \in\left[\tau_{1}\right]\right\}=\sup \left\{t:(t, j) \in\left[\tau_{2}\right]\right\}$ denote by $\left[\tau_{1}, \tau_{2}\right] \subset$ $\left[\tau_{1}\right] \times\left[\tau_{2}\right]$ any relation, satisfying the following conditions: $\left.\Pi\right|_{\left[\tau_{i}\right]}\left(\left[\tau_{1}, \tau_{2}\right]\right)=\left[\tau_{i}\right], i=1,2 ; \forall\left(\left(t_{a}, j_{a}\right),\left(t_{b}, j_{b}\right)\right) \in$ $\left[\tau_{1}, \tau_{2}\right], t_{a}=t_{b} ; \forall\left(\left(t_{a}, j_{a}\right),\left(t_{b}, j_{b}\right)\right),\left(\left(t_{a}^{\prime}, j_{a}^{\prime}\right),\left(t_{b}^{\prime}, j_{b}^{\prime}\right)\right) \in$ $\left[\tau_{1}, \tau_{2}\right]$, if $\left(t_{a}, j_{a}\right)<\left(t_{a}^{\prime}, j_{a}^{\prime}\right)$ then $\left(t_{b}, j_{b}\right)<\left(t_{b}^{\prime}, j_{b}^{\prime}\right)$ and vice versa; the last condition ensures that any relation $\left[\tau_{1}, \tau_{2}\right]$ preserves the ordering relation $<$ into each hybrid time basis $\left[\tau_{1}\right]$ and $\left[\tau_{2}\right]$.

Given two sets $Z_{1}, Z_{2}$, denote by $\mathcal{C}\left(Z_{1}, Z_{2}\right)$ and by $\mathcal{C}^{0}\left(Z_{1}, Z_{2}\right)$ the class of functions and respectively of piecewise continuous functions $z: Z_{1} \rightarrow Z_{2}$. The switching system temporal evolution may be now formally defined.

Definition 2: An execution $\chi$ of an SLS $\mathbb{S}$ is a collection $\left(\xi_{0}, \tau, u, d, \xi, y\right)$ with $\xi_{0}=\left(q_{0}, x_{0}\right) \in \Xi, \tau \in$ $\mathcal{T}, u \in \mathcal{C}^{0}\left(\mathbb{R}_{0}^{+}, U\right), d=(v, w)$, where $v \in \mathcal{C}(\mathbb{N}, V)$, $w \in \mathcal{C}^{0}\left(\mathbb{R}_{0}^{+}, W\right), \xi=(q, x)$, where $q \in \mathcal{C}(\mathbb{N}, Q), x \in$ $\mathcal{C}\left(\mathbb{R}_{0}^{+} \times \mathbb{N}, X().\right), y=(p, h)$, where $p \in \mathcal{C}(\mathbb{N}, P), h \in$ $\mathcal{C}\left(\mathbb{R}_{0}^{+} \times \mathbb{N}, H\right)$, such that, by setting $\xi(t, j)=(q(j), x(t, j))$ and $y(t, j)=(p(j), h(t, j))$, for all $(t, j) \in[\tau]$, the following conditions are satisfied: $q(j)=q_{0} ; q(j+1)$ is such that $e_{j}=(q(j), v(j+1), q(j+1)) \in E ; p(j)=$ $\gamma(q(j)) ; x\left(t_{0}, 0\right)=x_{0}, x\left(t_{j+1}, j+1\right)=M\left(e_{j+1}\right) x\left(t_{j}^{\prime}, j\right)$; moreover $x(t, j)$ and $h(t, j)$, for all $t \in I_{j}$ are respectively the unique solution and output at time $t$ of the dynamical system $\Sigma(q(j))$, with initial state $x\left(t_{j}, j\right)$, initial time $t_{j}$, input function $u^{\prime}$ and disturbance function $w^{\prime}$ such that, $u^{\prime}(t)=u\left(t-t_{j}\right)$ and $w^{\prime}(t)=w\left(t-t_{j}\right)$ for all $t \geq t_{j}$.

We now propose the notions of bisimulation and simulation for SLSs, obtained by merging the classical notions for concurrent processes (e.g. [4]) with new definitions introduced in [15], [16] for linear dynamical systems.

Definition 3: Given two SLSs $\mathbb{S}_{i}=\left(\Xi_{i}, U_{i}, D_{i}, Y_{i}, \Sigma_{i}\right.$, $\left.\gamma_{i}, E_{i}, M_{i}\right), i=1,2$ such that $U_{1}=U_{2}$, a hybrid bisimulation between $\mathbb{S}_{1}$ and $\mathbb{S}_{2}$ is a subset $\mathcal{R} \subset \Xi_{1} \times \Xi_{2}$ such that $\forall\left(\xi_{10}, \xi_{20}\right) \in \mathcal{R}$ and $\forall u_{1}=u_{2}$ the following property holds. For any $d_{1}$ and for any $\chi_{1}=\left(\xi_{10}, \tau_{1}, u_{1}, d_{1}, \xi_{1}, y_{1}\right)$ of $\mathbb{S}_{1}$, there exists $d_{2}$ and $\chi_{2}=\left(\xi_{20}, \tau_{2}, u_{2}, d_{2}, \xi_{2}, y_{2}\right)$ of $\mathbb{S}_{2}$ such that $\left(\xi_{1}(t, j), \xi_{2}\left(t^{\prime}, j^{\prime}\right)\right) \in \mathcal{R}$ and $y_{1}(t, j)=y_{2}\left(t^{\prime}, j^{\prime}\right)$, $\forall\left((t, j),\left(t^{\prime}, j^{\prime}\right)\right) \in\left[\tau_{1}, \tau_{2}\right]$, for some $\left[\tau_{1}, \tau_{2}\right]$. Moreover the same holds with $d_{1}$ replaced by $d_{2}$ and vice versa.

Definition 4: $\mathbb{S}_{1}$ and $\mathbb{S}_{2}$ are bisimilar, and we write $\mathbb{S}_{1} \sim$ $\mathbb{S}_{2}$, if there exists a hybrid bisimulation $\mathcal{R} \subset \Xi_{1} \times \Xi_{2}$ such that $\left.\Pi\right|_{\Xi_{i}}(\mathcal{R})=\Xi_{i}, i=1,2$.

Definition 5: Given two SLSs $\mathbb{S}_{i}=\left(\Xi_{i}, U_{i}, D_{i}, Y_{i}, \Sigma_{i}\right.$, $\left.\gamma_{i}, E_{i}, M_{i}\right), i=1,2$ such that $U_{1} \subset U_{2}$, a hybrid simulation between $\mathbb{S}_{1}$ and $\mathbb{S}_{2}$ is a subset $\mathcal{R} \subset \Xi_{1} \times \Xi_{2}$ such that $\forall\left(\xi_{10}, \xi_{20}\right) \in \mathcal{R}$ and $\forall u_{1}=u_{2}$ the following property holds. For any $d_{1}$ and for any $\chi_{1}=\left(\xi_{10}, \tau_{1}, u_{1}, d_{1}, \xi_{1}, y_{1}\right)$ of $\mathbb{S}_{1}$, there exists $d_{2}$ and $\chi_{2}=\left(\xi_{20}, \tau_{2}, u_{2}, d_{2}, \xi_{2}, y_{2}\right)$ of $\mathbb{S}_{2}$ such that $\left(\xi_{1}(t, j), \xi_{2}\left(t^{\prime}, j^{\prime}\right)\right) \in \mathcal{R}$ and $y_{1}(t, j)=y_{2}\left(t^{\prime}, j^{\prime}\right)$, $\forall\left((t, j),\left(t^{\prime}, j^{\prime}\right)\right) \in\left[\tau_{1}, \tau_{2}\right]$, for some $\left[\tau_{1}, \tau_{2}\right]$.

Definition 6: $\mathbb{S}_{1}$ is simulated by $\mathbb{S}_{2}$ (or equivalently $\mathbb{S}_{2}$ simulates $\mathbb{S}_{1}$ ), and we write $\mathbb{S}_{2} \rightsquigarrow \mathbb{S}_{1}$, if there exists a hybrid simulation $\mathcal{R} \subset \Xi_{1} \times \Xi_{2}$ of $\mathbb{S}_{1}$ by $\mathbb{S}_{2}$ such that $\left.\Pi\right|_{\Xi_{1}}(\mathcal{R})=\Xi_{1}$.

Bisimilarity between SLSs is an equivalence relation on the space of SLSs. Hybrid simulation is reflexive, transitive but not symmetric and hence it is not an equivalence relation on the space of SLSs. An equivalence notion, based on hybrid simulations can be formalized as follows.

Definition 7: $\mathbb{S}_{1}$ and $\mathbb{S}_{2}$ are similar if $\mathbb{S}_{1} \rightsquigarrow \mathbb{S}_{2} \rightsquigarrow \mathbb{S}_{1}$.

\section{B. Equivalent Switching Linear Systems}

Aim of this section is to define some control system theory equivalence notions for the class of SLSs and then to compare them with bisimilarity and similarity. The following definitions extend well known concepts given for the class of linear dynamical systems [3] and DESs [4].

Definition 8: $\mathbb{S}_{1}$ and $\mathbb{S}_{2}$ are algebraically equivalent if there exists an invertible mapping $T_{\mathbb{Q}}: Q_{1} \rightarrow Q_{2}$ and for any $q_{1} \in Q_{1}$, invertible linear mappings $T_{q_{1}}: X_{1}\left(q_{1}\right) \rightarrow$ $X_{2}\left(T_{\mathbb{Q}}\left(q_{1}\right)\right)$ such that, $\gamma_{1}\left(q_{1}\right)=\gamma_{2}\left(T_{\mathbb{Q}}\left(q_{1}\right)\right), \forall q_{1} \in Q_{1}$; $\forall e_{1}=\left(q_{1}, v_{1}, q_{1}^{\prime}\right) \in E_{1}$, there exists $v_{2} \in V_{2}$ such that $e_{2}=\left(T_{\mathbb{Q}}\left(q_{1}\right), v_{2}, T_{\mathbb{Q}}\left(q_{1}^{\prime}\right)\right) \in E_{2}$ and vice versa; $\forall q_{1} \in$ $Q_{1}, \Sigma_{1}\left(q_{1}\right)$ and $\Sigma_{2}\left(T_{\mathbb{Q}}\left(q_{1}\right)\right)$ are algebraically equivalent [3] with transformation matrix $T_{q_{1}} ; M_{1}\left(e_{1}\right)=T_{q_{1}^{\prime}}^{-1} M_{2}\left(e_{2}\right) T_{q_{1}}$, $\forall e_{1}=\left(q_{1}, v_{1}, q_{1}^{\prime}\right) \in E_{1}$ where $e_{2}=\left(T_{\mathbb{Q}}\left(q_{1}\right), v_{2}, T_{\mathbb{Q}}\left(q_{1}^{\prime}\right)\right) \in$ $E_{2}$, for some $v_{2} \in V_{2}$, and vice versa.

Definition 9: Given $\mathbb{S}_{1}$ and $\mathbb{S}_{2}$ with $U_{1}=U_{2}, \xi_{1} \in \Xi_{1}$ and $\xi_{2} \in \Xi_{2}$ are said to be state-equivalent if for any $u$, for any $d_{1}$, for any $\chi_{1}=\left(\xi_{10}, \tau_{1}, u, d_{1}, \xi_{1}, y_{1}\right)$ of $\mathbb{S}_{1}$, there exists $d_{2}$ and $\chi_{2}=\left(\xi_{20}, \tau_{2}, u, d_{2}, \xi_{2}, y_{2}\right)$ of $\mathbb{S}_{2}$ such that $y_{1}(t, j)=y_{2}\left(t^{\prime}, j^{\prime}\right), \forall\left((t, j),\left(t^{\prime}, j^{\prime}\right)\right) \in\left[\tau_{1}, \tau_{2}\right]$, for some $\left[\tau_{1}, \tau_{2}\right]$. Moreover the same holds with $d_{1}$ replaced by $d_{2}$ and vice versa.

Definition 10: $\mathbb{S}_{1}$ and $\mathbb{S}_{2}$ are state-space equivalent if $U_{1}=U_{2}$ and for any $\xi_{1} \in \Xi_{1}$ of $\mathbb{S}_{1}$, there exists $\xi_{2} \in \Xi_{2}$ of $\mathbb{S}_{2}$, equivalent to $\xi_{1}$ and vice versa.

Definition 11: $\mathbb{S}_{1}$ and $\mathbb{S}_{2}$ are Input-Output (I/O) equivalent if $U_{1}=U_{2}$ and for any $\xi_{10} \in \Xi_{1}$, for any $u \in U_{1}$, for any $d_{1}$, for any $\chi_{1}=\left(\xi_{10}, \tau_{1}, u, d_{1}, \xi_{1}, y_{1}\right)$ of $\mathbb{S}_{1}$, there exist $\xi_{20} \in \Xi_{2}, d_{2}$ and $\chi_{2}=\left(\xi_{20}, \tau_{2}, u, d_{2}, \xi_{2}, y_{2}\right)$ of $\mathbb{S}_{2}$ such that $y_{1}(t, j)=y_{2}\left(t^{\prime}, j^{\prime}\right), \forall\left((t, j),\left(t^{\prime}, j^{\prime}\right)\right) \in\left[\tau_{1}, \tau_{2}\right]$, for some $\left[\tau_{1}, \tau_{2}\right]$. Moreover the same holds with $\xi_{10}$ and $d_{1}$ replaced by $\xi_{20}$ and $d_{2}$ and vice versa.

Algebraic, state-space and input-output equivalences are equivalence relations on the space of SLSs. Moreover the following result holds.

Theorem 1: Two algebraically equivalent SLSs are bisimilar. Two bisimilar SLSs are similar. Two bisimilar SLSs are state-space equivalent. Two similar SLSs are input-output equivalent. Two state-space equivalent SLSs are input-output equivalent. 
Converse implications of the result above are not true in general but are true for deterministic SLSs (hybrid disturbance void) as remarked in [13].

\section{Characterizing Hybrid Bisimulations of SLSs}

The semantics of SLSs allow a complete characterization of hybrid bisimulations for SLSs as this section shows.

Proposition 2: If $\mathcal{R}$ is a hybrid bisimulation between two SLSs $\mathbb{S}_{1}$ and $\mathbb{S}_{2}$, there exists $Q_{\mathcal{R}} \subset Q_{1} \times Q_{2}$ and $\forall\left(q_{1}, q_{2}\right) \in$ $Q_{\mathcal{R}}$ suitable sets $\mathcal{R}\left(q_{1}, q_{2}\right) \subset X_{1}\left(q_{1}\right) \times X_{2}\left(q_{2}\right)$ such that $\left(\left(q_{1}, x_{1}\right),\left(q_{2}, x_{2}\right)\right) \in \mathcal{R}$ iff $\left(q_{1}, q_{2}\right) \in Q_{\mathcal{R}}$ and $\left(x_{1}, x_{2}\right) \in$ $\mathcal{R}\left(q_{1}, q_{2}\right)$.

Moreover if $\mathcal{R}\left(q_{1}, q_{2}\right)$ is a bisimulation relation between $\Sigma_{1}\left(q_{1}\right)$ and $\Sigma_{2}\left(q_{2}\right)$ then the linear closure [9] of the $\mathcal{R}\left(q_{1}, q_{2}\right)$ is still a bisimulation relation between $\Sigma_{1}\left(q_{1}\right)$ and $\Sigma_{2}\left(q_{2}\right)$. Therefore w.l.o.g. from now on, any hybrid bisimulation $\mathcal{R}$ between two SLSs $\mathbb{S}_{1}$ and $\mathbb{S}_{2}$ will be represented as:

$$
\left\{\left(\left(q_{1}, x_{1}\right),\left(q_{2}, x_{2}\right)\right) \mid\left(q_{1}, q_{2}\right) \in Q_{\mathcal{R}},\left(x_{1}, x_{2}\right) \in \mathcal{R}\left(q_{1}, q_{2}\right)\right\}
$$

where $Q_{\mathcal{R}} \subset Q_{1} \times Q_{2}$ and $\mathcal{R}\left(q_{1}, q_{2}\right)$ is a linear subspace of $X_{1}\left(q_{1}\right) \times X_{2}\left(q_{2}\right), \forall\left(q_{1}, q_{2}\right) \in Q_{\mathcal{R}}$. The following result gives an algebraic characterization of hybrid bisimulations for SLSs.

Theorem 3: Given $\mathbb{S}_{1}$ and $\mathbb{S}_{2}$, a set $\mathcal{R}$ of the form (1) is a hybrid bisimulation between $\mathbb{S}_{1}$ and $\mathbb{S}_{2}$ iff $\forall\left(q_{1}, q_{2}\right) \in$ $Q_{\mathcal{R}}$ the following property holds: $\forall q_{1}^{\prime} \in \operatorname{succ}\left(q_{1}\right), \exists q_{2}^{\prime} \in$ $\operatorname{succ}\left(q_{2}\right) \cup\left\{q_{2}\right\}:\left(q_{1}^{\prime}, q_{2}^{\prime}\right) \in Q_{\mathcal{R}}$ and

(3,i) $\gamma_{1}\left(q_{1}\right)=\gamma_{2}\left(q_{2}\right)$ and $\mathcal{R}\left(q_{1}, q_{2}\right)$ is a bisimulation relation between $\Sigma_{1}\left(q_{1}\right)$ and $\Sigma_{2}\left(q_{2}\right)$;

(3,ii) $\operatorname{diag}\left(M_{1}\left(e_{1}\right), \bar{M}_{2}\right) \mathcal{R}\left(q_{1}, q_{2}\right) \subset \mathcal{R}\left(q_{1}^{\prime}, q_{2}^{\prime}\right)$, where $e_{1} \in E_{1}$ takes $q_{1}$ into $q_{1}^{\prime}$ and $e_{2} \in E_{2}$ takes $q_{2}$ into $q_{2}^{\prime}$, and $\bar{M}_{2}=M_{2}\left(e_{2}\right)$ if $q_{2}^{\prime} \neq q_{2}, \bar{M}_{2}=I$ if $q_{2}^{\prime}=q_{2}$; and vice versa, $\forall q_{2}^{\prime} \in \operatorname{succ}\left(q_{2}\right), \exists q_{1}^{\prime} \in \operatorname{succ}\left(q_{1}\right) \cup\left\{q_{1}\right\}$ : $\left(q_{1}^{\prime}, q_{2}^{\prime}\right) \in Q_{\mathcal{R}}$ and conditions $(3, \mathrm{i})$ and $(3, \mathrm{ii})$ are satisfied.

A consequence of Theorem 3 is given in the following.

Corollary 4: $\mathbb{S}_{1}$ and $\mathbb{S}_{2}$ are bisimilar iff there exists a set $\mathcal{R} \subset \Xi_{1} \times \Xi_{2}$ of the form (1) satisfying conditions of Theorem 3 and such that $\left.\Pi\right|_{\Xi_{i}}(\mathcal{R})=\Xi_{i}, i=1,2$.

Finally the following holds.

Proposition 5: Let $\mathbb{S}_{1}$ and $\mathbb{S}_{2}$ be bisimilar and let $\mathcal{R}$ be a hybrid bisimulation between $\mathbb{S}_{1}$ and $\mathbb{S}_{2}$ such that $\left.\Pi\right|_{\Xi_{i}}(\mathcal{R})=\Xi_{i}, i=1,2$. For any $q_{1} \in Q_{1}$, there exists $q_{2} \in Q_{2}$ such that $\Sigma_{1}\left(q_{1}\right) \sim \Sigma_{2}\left(q_{2}\right)$ and $\left(q_{1}, q_{2}\right) \in Q_{\mathcal{R}}$ and vice versa; for any $\left(q_{1}, q_{2}\right) \in Q_{\mathcal{R}}, \Sigma_{1}\left(q_{1}\right) \sim \Sigma_{2}\left(q_{2}\right)$ or $\Sigma_{1}\left(q_{1}\right) \rightsquigarrow \Sigma_{2}\left(q_{2}\right)$ or $\Sigma_{2}\left(q_{2}\right) \rightsquigarrow \Sigma_{1}\left(q_{1}\right)$.

\section{MAXimal HyBRid Bisimulation}

The maximal hybrid bisimulation between bisimilar SLSs $\mathbb{S}_{1}$ and $\mathbb{S}_{2}$ is a hybrid bisimulation $\mathcal{R}^{*}$ such that, for all hybrid bisimulations $\mathcal{R}, \mathcal{R} \subset \mathcal{R}^{*}$. In order to prove the existence of $\mathcal{R}^{*}$ we define the sum $\mathcal{R}^{a b}$ of two hybrid bisimulations $\mathcal{R}^{a}$ and $\mathcal{R}^{b}$ between two bisimilar SLSs $\mathbb{S}_{1}$ and $\mathbb{S}_{2}$ as

$$
\begin{gathered}
\mathcal{R}^{a b}:=\mathcal{R}^{a}+\mathcal{R}^{b}=\left\{\left(\left(q_{1}, x_{1}\right),\left(q_{2}, x_{2}\right)\right) \in \Xi_{1} \times \Xi_{2} \mid\right. \\
\left.\left(q_{1}, q_{2}\right) \in Q_{\mathcal{R}^{a b}},\left(x_{1}, x_{2}\right) \in \mathcal{R}^{a b}\left(q_{1}, q_{2}\right\}\right),
\end{gathered}
$$

where $Q_{\mathcal{R}^{a b}}:=Q_{\mathcal{R}^{a}} \cup Q_{\mathcal{R}^{b}}$ and $\mathcal{R}^{a b}\left(q_{1}, q_{2}\right):=$

$$
\begin{cases}\mathcal{R}^{a}\left(q_{1}, q_{2}\right)+\mathcal{R}^{b}\left(q_{1}, q_{2}\right), & \text { if }\left(q_{1}, q_{2}\right) \in Q_{\mathcal{R}^{a}} \cap Q_{\mathcal{R}^{b}} \\ \mathcal{R}^{a}\left(q_{1}, q_{2}\right), & \text { if }\left(q_{1}, q_{2}\right) \in Q_{\mathcal{R}^{a}} \backslash Q_{\mathcal{R}^{b}} \\ \mathcal{R}^{b}\left(q_{1}, q_{2}\right), & \text { if }\left(q_{1}, q_{2}\right) \in Q_{\mathcal{R}^{b}} \backslash Q_{\mathcal{R}^{a}}\end{cases}
$$

Proposition 6: Let $\mathcal{R}^{a}$ and $\mathcal{R}^{b}$ be hybrid bisimulations between bisimilar SLSs $\mathbb{S}_{1}$ and $\mathbb{S}_{2}$. Then $\mathcal{R}^{a}+\mathcal{R}^{b}$ is a hybrid bisimulation between $\mathbb{S}_{1}$ and $\mathbb{S}_{2}$.

This immediately ensures the existence of the maximal hybrid bisimulation:

Proposition 7: Let $\mathbb{S}_{1}$ and $\mathbb{S}_{2}$ be two SLSs and let $\mathcal{R} \neq$ $\varnothing$ be a hybrid bisimulation between $\mathbb{S}_{1}$ and $\mathbb{S}_{2}$, then the maximal hybrid bisimulation exists.

The key idea is to first compute the maximal bisimulation $Q^{*}$ between $\mathbb{D}_{\mathbb{S}_{1}}$ and $\mathbb{D}_{\mathbb{S}_{2}}$ and then, to compute $\mathcal{R}^{*}$ on the basis of $Q^{*}$. The computation of $Q^{*}$ may be performed by using standard algorithms as for example the one in [4] which converges in a finite number of steps to the maximal bisimulation relation for DESs. Note that $Q_{\mathcal{R}^{*}} \subset Q^{*}$.

The computation of $\mathcal{R}^{*}$ may be done by combining Algorithm 2 of [15], computing the maximal bisimulation and/or simulation relation for linear dynamical systems, with Procedure Switching of [5] for the computation of maximal safe sets for switching systems. Computing $\mathcal{R}^{*}$ requires the analysis of the topological properties of DESs $\mathbb{D}_{\mathbb{S}_{1}}$ and $\mathbb{D}_{\mathbb{S}_{2}}$ associated to SLSs $\mathbb{S}_{1}$ and $\mathbb{S}_{2}$. For this purpose, it is useful to define the DES $\mathbb{D}^{*}=\left(Q^{*}, P^{*}, V^{*}, E^{*}, \gamma^{*}\right)$, naturally induced by the bisimulation relation $Q^{*}$, where

- $P^{*}=P_{1} \cup P_{2}$;

- $V^{*}=V_{1} \times V_{2}$

- $E^{*}=\left\{\left(\left(q_{1}, q_{2}\right),\left(v_{1}, v_{2}\right),\left(q_{1}^{\prime}, q_{2}^{\prime}\right)\right):\left(q_{1}, v_{1}, q_{1}^{\prime}\right) \in E_{1}\right.$, $\left.\left(q_{2}, v_{2}, q_{2}^{\prime}\right) \in E_{2}, \gamma_{1}\left(q_{1}\right)=\gamma_{2}\left(q_{2}\right), \gamma_{1}\left(q_{1}^{\prime}\right)=\gamma_{2}\left(q_{2}^{\prime}\right)\right\}$;

- $\gamma^{*}\left(q_{1}, q_{2}\right)=\gamma_{1}\left(q_{1}\right)=\gamma_{2}\left(q_{2}\right), \forall\left(q_{1}, q_{2}\right) \in Q^{*}$.

Before explaining the basic steps of the proposed procedure for the computation of $\mathcal{R}^{*}$, we need to recall well known facts about DESs [8]. A Strongly Connected Component (SCC) of $\mathbb{D}^{*}$ is the maximal set of mutually reachable states. We denote by $\mathbf{F}$, the set of all SCCs associated to $\mathbb{D}^{*}$. SCCs determine a Directed Acyclic Graph (DAG). Moreover $\mathbf{F}^{0} \subset \mathbf{F}$ denotes the set of all SCCs not reached by any SCCs, $\mathbf{F}^{i} \subset \mathbf{F}$ denotes the set of all SCCs, reached in one step by a SCC in $\mathbf{F}^{i-1}$ and so on. Let $n_{\mathbf{F}}$ be the maximal integer $i$ for which $\mathbf{F}^{i}$ is nonempty. Any SCC in $\mathbf{F}^{0}$ (resp. in $\mathbf{F}^{n_{F}}$ ) is called a root (resp. a leaf) of the DAG associated to $\mathbb{D}^{*}$. For any $F \in \mathbf{F}$, we denote by $Q_{F} \subset$ $Q_{1} \times Q_{2}$, the set of extended discrete states belonging to $F$. Moreover $\forall\left(q_{1}, q_{2}\right) \in Q_{F}$ for some $F, \operatorname{succ}\left(q_{1}, q_{2}\right)$ denotes the set of all extended discrete states that are successor of $\left(q_{1}, q_{2}\right)$ in $F$. For any $F$ of $\mathbb{D}^{*}$, the set $\operatorname{succ}(F)$ is composed by those SCCs, reached by $F$ in one step and $\mathbb{S}_{i}(F)$, $i=1,2$ denote the pair of bisimilar SLSs naturally induced by the DES $\mathbb{D}^{*}$ and by continuous dynamics associated to its extended discrete states. $\mathcal{G}\left(F^{+}, F^{-}\right) \subset Q_{F^{+}}$denotes the set of extended discrete states of $F^{+}$, reachable in one step by an extended discrete state in $F^{-}$. Moreover 
$M^{\dagger}$ denotes the Moore-Penrose pseudo-inverse of a given matrix $M$. The computation of $\mathcal{R}^{*}$ is based on Theorem 3 and is carried out using a two step procedure: at the lower-level, we give a procedure for the computation of the maximal hybrid bisimulation between bisimilar SLSs $\mathbb{S}_{1}(F)$ and $\mathbb{S}_{2}(F)$, constrained to a given subspace $\operatorname{Init}(F)$; at the higher-level, the computation of $\mathcal{R}^{*}$ is proposed and based on the lower-level procedure. In view of Proposition 5, for any pair $\Sigma_{1}\left(q_{1}\right)$ and $\Sigma_{2}\left(q_{2}\right)$ such that $\left(q_{1}, q_{2}\right) \in Q^{*}$ denote by $\operatorname{rel}\left(\Sigma_{1}\left(q_{1}\right), \Sigma_{2}\left(q_{2}\right)\right.$, Init) a function computing the maximal linear bisimulation of $\Sigma_{1}\left(q_{1}\right)$ and $\Sigma_{2}\left(q_{2}\right)$ (resp. simulation of $\Sigma_{1}\left(q_{1}\right)$ by $\Sigma_{2}\left(q_{2}\right)$, simulation of $\Sigma_{2}\left(q_{2}\right)$ by $\Sigma_{1}\left(q_{1}\right)$ ) constrained to Init, if $\Sigma_{1}\left(q_{1}\right) \sim \Sigma_{2}\left(q_{2}\right)$ (resp. if $\Sigma_{1}\left(q_{1}\right) \rightsquigarrow \Sigma_{2}\left(q_{2}\right)$, if $\left.\Sigma_{2}\left(q_{2}\right) \rightsquigarrow \Sigma_{1}\left(q_{1}\right)\right)$ on the basis of procedures of [15]. We start by describing how to compute the maximal hybrid bisimulation $\operatorname{BisimSCC}(\operatorname{Init}(F), F)$ between SLSs $\mathbb{S}_{1}(F)$ and $\mathbb{S}_{2}(F)$ induced by the SCC $F$ of $\mathbb{D}^{*}$ and constrained in the hybrid subspace $\operatorname{Init}(F)=$ $\bigcup_{\left(q_{1}, q_{2}\right) \in Q_{F}}\left\{\left(q_{1}, q_{2}\right)\right\} \times \operatorname{Init}^{F}\left(q_{1}, q_{2}\right)$. We will define an appropriate recursion which, by exploiting topological structure of $F$, computes a sequence of sets $K(i), i=$ $0,1, \ldots$, converging to the maximal hybrid bisimulation between $\mathbb{S}_{1}(F)$ and $\mathbb{S}_{2}(F)$. At first we set $i=0$ and the initial maximal hybrid bisimulation $K(0)$ between $\mathbb{S}_{1}(F)$ and $\mathbb{S}_{2}(F)$ as $K(0):=\bigcup_{\left(q_{1}, q_{2}\right) \in Q_{F}}\left\{\left(q_{1}, q_{2}\right)\right\} \times Z\left(\left(q_{1}, q_{2}\right), 0\right)$, where $Z\left(\left(q_{1}, q_{2}\right), 0\right):=\operatorname{Init}^{F}\left(q_{1}, q_{2}\right)$ for any $\left(q_{1}, q_{2}\right) \in$ $Q_{F}$. For any $\left(q_{1}, q_{2}\right) \in Q_{F}$, we first update the constraining subspace $Z_{0}$ where $\operatorname{rel}\left(\Sigma_{1}\left(q_{1}\right), \Sigma_{2}\left(q_{2}\right), Z_{0}\right)$ lies: for any $\left(q_{1}^{\prime}, q_{2}^{\prime}\right) \in \operatorname{succ}\left(q_{1}, q_{2}\right)$, thanks to condition (3,ii) of Theorem $3, \operatorname{rel}\left(\Sigma_{1}\left(q_{1}\right), \Sigma_{2}\left(q_{2}\right), Z_{0}\right)$ has to belong to $M^{\dagger}\left(e_{1}, e_{2}\right) Z\left(\left(q_{1}^{\prime}, q_{2}^{\prime}\right), 0\right)$, where $e_{1}$ and $e_{2}$ connect discrete states $\left(q_{1}, q_{2}\right)$ to $\left(q_{1}^{\prime}, q_{2}^{\prime}\right)$. Compute $Z_{0}:=$ $\bigcap_{\left(q_{1}^{\prime}, q_{2}^{\prime}\right) \in \operatorname{succ}\left(q_{1}, q_{2}\right)} M^{\dagger}\left(e_{1}, e_{2}\right) Z\left(\left(q_{1}^{\prime}, q_{2}^{\prime}\right), 0\right)$. Then it is possible to compute $Z\left(\left(q_{1}, q_{2}\right), 0\right):=\operatorname{rel}\left(\Sigma_{1}\left(q_{1}\right), \Sigma_{2}\left(q_{2}\right), Z_{0}\right)$ between $\Sigma_{1}\left(q_{1}\right)$ and $\Sigma_{2}\left(q_{2}\right)$. Finally we update $K(0):=$ $\bigcup_{\left(q_{1}, q_{2}\right) \in Q_{F}}\left\{\left(q_{1}, q_{2}\right)\right\} \times Z\left(\left(q_{1}, q_{2}\right), 0\right)$ and $i:=i+1$. By iterating this step again the maximal hybrid bisimulation between $\mathbb{S}_{1}(F)$ and $\mathbb{S}_{2}(F)$ corresponds to a fixed point $K(i)=K(i-1)$, for some $i \in \mathbb{N}$, of the recursion above. The proposed approach is formalized in the following Function.

$$
\begin{aligned}
& \text { Function } 8: R(F):=\operatorname{BisimSCC}(\operatorname{Init}(F), F) \\
& \text { set } i:=0 \\
& \forall\left(q_{1}, q_{2}\right) \in Q_{F} \text {, set } Z\left(\left(q_{1}, q_{2}\right), i\right):=\operatorname{Init}{ }^{F}\left(q_{1}, q_{2}\right) \\
& \text { set } K(i):=\bigcup_{\left(q_{1}, q_{2}\right) \in Q_{F}}\left\{\left(q_{1}, q_{2}\right)\right\} \times Z\left(\left(q_{1}, q_{2}\right), i\right) \\
& \text { while } K(i) \neq=(i-1) \text { repeat } \\
& \text { for any }\left(q_{1}, q_{2}\right) \in Q_{F} \text { do } \\
& \text { compute } Z_{0}:=\bigcap_{\left(q_{1}^{\prime}, q^{\prime}\right) \in \operatorname{succ}\left(q_{1}, q_{2}\right)} M^{\dagger}\left(e_{1}, e_{2}\right) Z\left(\left(q_{1}^{\prime}, q_{2}^{\prime}\right), i\right) \\
& \text { where } e_{k}=\left(q_{k}, v_{k}, q_{k}^{\prime}\right), v_{k} \in V_{k}, k=1,2 \\
& \text { compute } Z\left(\left(q_{1}, q_{2}\right), i\right)=\operatorname{rel}\left(\Sigma_{1}\left(q_{1}\right), \Sigma_{2}\left(q_{2}\right), Z_{0}\right) \\
& \text { end do } \\
& \text { set } K(i):=\bigcup_{\left(q_{1}, q_{2}\right) \in Q_{F}}\left\{\left(q_{1}, q_{2}\right)\right\} \times Z\left(\left(q_{1}, q_{2}\right), i\right) \\
& \text { set } i:=i+1 \\
& \text { end while } \\
& \text { return } R(F):=K(i) \\
& \text { end Function }
\end{aligned}
$$

We can now provide the high-level algorithm. The computation of the maximal hybrid bisimulation starts from the leaves $F \in \mathbf{F}^{n_{\mathbf{F}}}$ and going backwards, ends to the roots $F \in \mathbf{F}^{0}$ of the DAG associated to $\mathbb{D}^{*}$. For any $F \in \mathbf{F}$, Init $(F)$ represents the constraining subspace where the maximal hybrid bisimulation has to be computed. Firstly we set $\operatorname{Init}(F):=\bigcup_{\left(q_{1}, q_{2}\right) \in Q_{F}}\left\{\left(q_{1}, q_{2}\right)\right\} \times \operatorname{Init}^{F}\left(q_{1}, q_{2}\right)$, $\forall F \in \mathbf{F}$. Note that any $F \in \mathbf{F}^{n_{\mathbf{F}}}$ has no successors. The first step consists of computing for any $F \in \mathbf{F}^{n_{\mathbf{F}}}$, the maximal hybrid bisimulation $\operatorname{BisimSCC}(\operatorname{Init}(F), F)$ associated to SLSs $\mathbb{S}_{1}(F)$ and $\mathbb{S}_{2}(F)$, induced by $F$. Then we need to update the constraining subspace $\operatorname{Init}\left(F^{-}\right)$ of SCCs $F^{-} \in \operatorname{succ}^{-1}\left(\mathbf{F}^{n_{\mathbf{F}}}\right)$ : consider all SCCs $F^{+} \in$ $\operatorname{succ}\left(F^{-}\right)$and all extended discrete states $\left(q_{1}, q_{2}\right) \in$ $Q_{F^{-}}$that reach in one step the extended discrete states $\left(q_{1}^{\prime}, q_{2}^{\prime}\right) \in \mathcal{G}\left(F^{+}, F^{-}\right) \subset Q_{F^{+}}$of $F^{+}$. By condition (3,ii), for any fixed $F^{+} \in \operatorname{succ}\left(F^{-}\right)$and for any fixed $\left(q_{1}^{\prime}, q_{2}^{\prime}\right) \in \mathcal{G}\left(F^{+}, F^{-}\right)$, the set $\operatorname{rel}\left(\left(q_{1}, q_{2}\right), Z_{0}\right)$ has to belong to $\alpha\left(q_{1}^{\prime}, q_{2}^{\prime}\right):=M^{\dagger}\left(e_{1}, e_{2}\right) \operatorname{rel}\left(\left(q_{1}^{\prime}, q_{2}^{\prime}\right)\right.$, Init $\left.\left(F^{+}\right)\right)$, where $e_{1}$ and $e_{2}$ connect $\left(q_{1}, q_{2}\right) \in Q_{F^{-}}$to $\left(q_{1}^{\prime}, q_{2}^{\prime}\right) \in$ $Q_{F^{+}}$. By considering all extended discrete states $\left(q_{1}^{\prime}, q_{2}^{\prime}\right) \in$ $\mathcal{G}\left(F^{+}, F^{-}\right)$and all SCCs $F^{+} \in \operatorname{succ}\left(F^{-}\right)$, the maximal hybrid bisimulation between $\mathbb{S}_{1}\left(F^{-}\right)$and $\mathbb{S}_{2}\left(F^{-}\right)$, has to belong to $\operatorname{Init}\left(F^{-}\right):=\operatorname{Init}\left(F^{-}\right) \bigcap I^{0}\left(F^{-}\right)$where $I^{0}\left(F^{-}\right):=\bigcap_{F^{+} \in \operatorname{succ}\left(F^{-}\right)}\left(\bigcap_{\left(q_{1}^{\prime}, q_{2}^{\prime}\right) \in \mathcal{G}\left(F^{+}, F^{-}\right)} \alpha\left(q_{1}^{\prime}, q_{2}^{\prime}\right)\right)$. Finally it is possible to go backwards and consider the SCCs of $\mathbf{F}^{n_{\mathbf{F}}-1}$ and so on. This procedure ends when all SCCs are visited. The proposed approach is formalized in the following Algorithm.

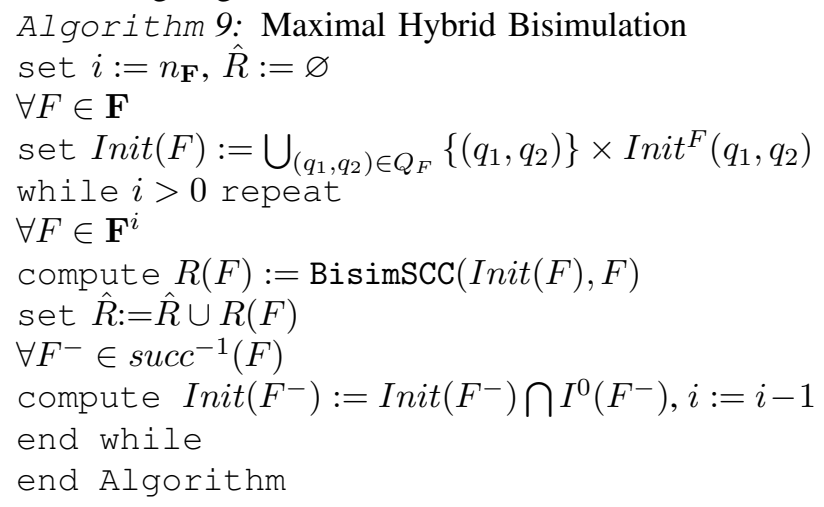

Proposition 10: Given a SCC $F$ and an initial subspace Init $(F)$, Function $\operatorname{BisimSCC}(\operatorname{Init}(F), F)$ converges in at most $\aleph(F)$ steps, where $\aleph(F)=\max \left\{\operatorname{dim} X_{1}\left(q_{1}\right)+\right.$ $\left.\operatorname{dim} X_{2}\left(q_{2}\right),\left(q_{1}, q_{2}\right) \in Q_{F}\right\}$. Algorithm 9 converges in at most $\aleph$ steps to $\hat{R}=\mathcal{R}^{*}$ or to the empty set, where $\aleph=\sum_{F \in \mathbf{F}} \aleph(F)$.

\section{Reduction VIa Hybrid Bisimulation}

In this section, we extend the results in [7] and [15] in order to reduce the complexity of SLSs. We therefore will consider an SLS $\mathbb{S}$ and a copy of itself and we show how to perform a hybrid state space reduction of $\mathbb{S}$. The following obvious facts hold:

Lemma 11: Given an SLS $\mathbb{S}$, the identity relation $\mathcal{R}_{i d}:=$ $\left\{\left(\xi_{1}, \xi_{2}\right) \mid \xi_{1}=\xi_{2}\right\}$ is a hybrid bisimulation between $\mathbb{S}$ and 
itself and for any hybrid bisimulation $\mathcal{R}$ between $\mathbb{S}$ and itself, $\mathcal{R}^{-1}:=\left\{\left(\xi_{2}, \xi_{1}\right) \mid\left(\xi_{1}, \xi_{2}\right) \in \mathcal{R}\right\}$ is a hybrid bisimulation between $\mathbb{S}$ and itself.

Every $\mathcal{R} \subset \Xi \times \Xi$ induces a relation on $\Xi$ by saying that $\xi_{1}, \xi_{2} \in \Xi$ are related by $\mathcal{R}$ iff $\left(\xi_{1}, \xi_{2}\right) \in \mathcal{R}$. For performing the hybrid state space reduction of $\mathbb{S}$, we need an equivalence relation on $\Xi$ such that, all hybrid states belonging to the same equivalence class induced by the equivalence relation, are reduced to the same hybrid state.

Proposition 12: For any hybrid bisimulation $\mathcal{R}$ between $\mathbb{S}$ and itself there exists a hybrid bisimulation $\mathcal{R}^{\prime}$ between $\mathbb{S}$ and itself that is also an equivalence relation on $\Xi$.

We therefore may assume w.l.o.g. that hybrid bisimulations are also equivalence relations on $\Xi$. Moreover it is easy to see that $\mathcal{R}^{*}$ between $\mathbb{S}$ and itself is also an equivalence relation. Given a hybrid bisimulation and equivalence relation $\mathcal{R}$, we now show how to perform a hybrid state space reduction and how to define the reduced SLS bisimilar to $\mathbb{S}$. Denote by $\Omega_{i}$, the equivalence class induced by $Q_{\mathcal{R}}$ such that $q_{j}, q_{k} \in \Omega_{i}$ iff $\left(q_{j}, q_{k}\right) \in Q_{\mathcal{R}}$. For any $\Omega_{i}$ choose its canonical representative $q_{i}^{*} \in Q$ such that $\Sigma\left(q_{i}^{*}\right) \rightsquigarrow \Sigma(q), \forall q \in \Omega_{i}$ and denote by $Q^{\mathcal{R}}$ the set of all canonical representatives of the sequence $\Omega_{i}, i=1,2, \ldots$. For any $q \in Q^{\mathcal{R}}$ define $\mathcal{E}(q):=\left\{q^{\prime} \in Q:\left(q, q^{\prime}\right) \in\right.$ $\left.Q_{\mathcal{R}}\right\}, \overline{\mathcal{R}}(q):=\left\{x_{1}-x_{2} \mid\left(x_{1}, x_{2}\right) \in \mathcal{R}(q, q)\right\}$ and finally $\overline{\mathcal{R}}:=\bigcup_{q \in Q^{\mathcal{R}}} \mathcal{E}(q) \times \overline{\mathcal{R}}(q)$. $\Xi$ may be now factorized by $\overline{\mathcal{R}}$. We write $\Xi / \overline{\mathcal{R}}$ to denote the reduced hybrid state space of $\mathbb{S}$, naturally induced by $\overline{\mathcal{R}}$, i.e. $\Xi /_{\overline{\mathcal{R}}}=\bigcup_{q \in Q^{\mathcal{R}}}\{q\} \times$ $X(q) / \overline{\mathcal{R}}(q)$. Let $\Pi_{\mathbb{Q}}^{\mathcal{R}}: Q \rightarrow Q^{\mathcal{R}}$ be the canonical projection map associating to each element of $Q$, its unique canonical representative in $Q^{\mathcal{R}}$ and $\forall q \in Q^{\mathcal{R}}$, let $\Pi_{q}^{\mathcal{R}}$ : $X(q) \rightarrow X(q) / \overline{\mathcal{R}}(q)$ be the canonical projection. Define the reduced $S L S \mathbb{S}^{\mathcal{R}}=\left(\Xi^{\mathcal{R}}, U, D, Y, \Sigma^{\mathcal{R}}, \gamma^{\mathcal{R}}, E^{\mathcal{R}}, M^{\mathcal{R}}\right)$, where $X^{\mathcal{R}}(q)=X(q) / \overline{\mathcal{R}}(q)$ and $\operatorname{dim}^{\mathcal{R}}(q)=\operatorname{dim}\left(X^{\mathcal{R}}(q)\right)$, $\forall q \in Q^{\mathcal{R}} ; \Sigma^{\mathcal{R}}(q)$ is defined as in [15], $\forall q \in Q^{\mathcal{R}}$; $\gamma^{\mathcal{R}}: Q^{\mathcal{R}} \rightarrow P$ such that $\gamma^{\mathcal{R}}(q)=\gamma(q), \forall q \in Q^{\mathcal{R}} ; e=$ $\left(q_{1}^{\prime}, v, q_{2}^{\prime}\right) \in E^{\mathcal{R}}$ iff $\exists q_{1} \in \mathcal{E}^{-1}\left(q_{1}^{\prime}\right), q_{2} \in \mathcal{E}^{-1}\left(q_{2}^{\prime}\right)$ such that $\left(q_{1}, v, q_{2}\right) \in E ; \forall e=\left(q, v, q^{\prime}\right) \in E^{\mathcal{R}}, M^{\mathcal{R}}(e) \Pi_{q^{\prime}}^{\mathcal{R}}=$ $\Pi_{q}^{\mathcal{R}} M(e)$. The reduced SLS $\mathbb{S}^{\mathcal{R}}$ actually depends on $Q^{\mathcal{R}}$, however the following holds:

Proposition 13: Let $\mathcal{R}$ be a hybrid bisimulation and equivalence relation between $\mathbb{S}$ and itself. Then for any canonical representative $Q^{\mathcal{R}}, \mathbb{S}$ and $\mathbb{S}^{\mathcal{R}}$ are bisimilar.

Let us investigate the reduction of a SLS to a SLS with minimal dimension. A minimal bisimilar SLS of $\mathbb{S}$ is a SLS $\mathbb{S}^{\prime}$ such that the cardinality of its discrete state space $Q^{\prime}$ and the dimensions of its continuous state space $X^{\prime}(q), q \in Q^{\prime}$, are minimal among all $\mathbb{S}^{\prime}$ which are bisimilar to $\mathbb{S}$. Denote by $\min (\mathbb{S})$ the class of minimal bisimilar SLSs of $\mathbb{S}$. The following result holds:

Corollary 14: $\mathbb{S}^{\mathcal{R}^{*}} \in \min (\mathbb{S})$.

Since $\mathcal{R}^{*}$ depends on the set $Q^{\mathcal{R}^{*}}$, then also $\mathbb{S}^{\mathcal{R}^{*}}$ depends on $Q^{\mathcal{R}^{*}}$ and therefore it is not unique; however the following result holds.

Proposition 15: The family of $\mathbb{S}^{*}$, parametrized by $Q^{\mathcal{R}^{*}}$, is composed of SLSs that are algebraically equivalent.

\section{Simulation and AbStraction}

Aim of this section is to characterize the notion of simulation and to introduce the notion of abstraction [1], [12], [14] for the class of SLSs. By specializing Theorem 3 , the following result is obtained.

Theorem 16: Given $\mathbb{S}_{1}$ and $\mathbb{S}_{2}, \mathcal{R}$ of the form (1) is a simulation of $\mathbb{S}_{1}$ by $\mathbb{S}_{2}$ iff $\forall\left(q_{1}, q_{2}\right) \in Q_{\mathcal{R}}, \forall q_{1}^{\prime} \in \operatorname{succ}\left(q_{1}\right)$, $\exists q_{2}^{\prime} \in \operatorname{succ}\left(q_{2}\right) \cup\left\{q_{2}\right\}:\left(q_{1}^{\prime}, q_{2}^{\prime}\right) \in Q_{\mathcal{R}}$ and

$\left(16\right.$,i) $\gamma_{1}\left(q_{1}\right)=\gamma_{2}\left(q_{2}\right)$ and $\mathcal{R}\left(q_{1}, q_{2}\right)$ is a simulation relation between $\Sigma_{1}\left(q_{1}\right)$ and $\Sigma_{2}\left(q_{2}\right)$;

(16,ii) $\operatorname{diag}\left(M_{1}\left(e_{1}\right), \bar{M}_{2}\right) \mathcal{R}\left(q_{1}, q_{2}\right) \quad \subset \mathcal{R}\left(q_{1}^{\prime}, q_{2}^{\prime}\right)$, where $e_{1} \in E_{1}$ takes $q_{1}$ into $q_{1}^{\prime}$ and $e_{2} \in E_{2}$ takes $q_{2}$ into $q_{2}^{\prime}$, and $\bar{M}_{2}=M_{2}\left(e_{2}\right)$ if $q_{2}^{\prime} \neq q_{2}, \bar{M}_{2}=I$ if $q_{2}^{\prime}=q_{2}$;

By specializing results of Section III, it is possible to give also a procedure for the computation of the maximal hybrid simulation $\mathbb{S}_{1}$ by $\mathbb{S}_{2}$ by replacing the algorithms computing the maximal bisimulation of DESs and of linear dynamical systems with the ones in [4] and [15], computing the maximal simulation of $\mathbb{D}_{1}$ by $\mathbb{D}_{2}$, and respectively of $\Sigma_{1}$ by $\Sigma_{2}$. The proposed procedure converges to the maximal simulation of $\mathbb{S}_{1}$ by $\mathbb{S}_{2}$ in a finite number of steps. The notion of simulation is very close to the notion of abstraction. The main idea is to "simplify" the given process such that the resulting process simulates the original one. Following [12], [15], it is possible also to define abstractions of SLSs. Consider a SLS $\mathbb{S}=(\Xi, U, D, Y, \Sigma, \gamma, E, M)$. Define a suitable set $\Xi^{\mathcal{A}}=\bigcup_{q \in Q^{\mathcal{A}}}\{q\} \times X^{\mathcal{A}}(q)$, such that $Q^{\mathcal{A}} \subset Q$ and $\gamma\left(Q^{\mathcal{A}}\right)=\gamma(Q)$ and for any $q \in Q^{\mathcal{A}}$, let $X^{\mathcal{A}}(q)$ be a linear subspace of $X(q)$. Define a surjective map $\mathcal{M}$ : $\Xi \rightarrow \Xi^{\mathcal{A}}$, such that $\mathcal{M}(q, x)=\left(\mathcal{M}_{\mathbb{Q}}(q), \mathcal{M}_{q} x\right), \forall(q, x) \in$ $\Xi$, where $\mathcal{M}_{\mathbb{Q}}: Q \rightarrow Q^{\mathcal{A}}$ and $\forall q \in Q, \mathcal{M}_{q} x$ is linear in $x$. Suppose that $\forall q \in Q$, $\operatorname{ker} \mathcal{M}_{q} \subset \operatorname{ker} C(q)$. Map $\mathcal{M}$ induces the SLS $\mathbb{S}^{\mathcal{A}}:=\left(\Xi^{\mathcal{A}}, U, D, Y, \Sigma^{\mathcal{A}}, \gamma^{\mathcal{A}}, E^{\mathcal{A}}, M^{\mathcal{A}}\right)$, where: $\forall q \in Q^{\mathcal{A}}, \Sigma^{\mathcal{A}}(q)$ is defined as in [15]; $\gamma^{\mathcal{A}}\left(q^{\prime}\right)=$ $\gamma\left(\mathcal{M}_{\mathbb{Q}}^{-1}(q)\right)$ for some $q \in Q ; e=\left(q_{1}^{\prime}, v, q_{2}^{\prime}\right) \in E^{\mathcal{A}}$ iff there exist $q_{1} \in \mathcal{M}_{\mathbb{Q}}^{-1}\left(q_{1}^{\prime}\right)$ and $q_{2} \in \mathcal{M}_{\mathbb{Q}}^{-1}\left(q_{2}^{\prime}\right)$ such that $\left(q_{1}, v, q_{2}\right) \in E ; M^{\mathcal{A}}(e)=\mathcal{M}_{q} M(e) \mathcal{M}_{q}^{\dagger}, \forall\left(q, w, q^{\prime}\right) \in E^{\mathcal{A}}$.

We think of $\mathbb{S}^{\mathcal{A}}$ as an abstraction of $\mathbb{S}$. Moreover,

Proposition 17: $\mathbb{S} \rightarrow \mathbb{S}^{\mathcal{A}}$.

\section{CONNECTIONS To OBSERVABILITy}

In this section, we analyze the preservation of observability under bisimulation-based reduction and simulationbased abstraction. The class of SLSs that we consider are SLSs with $W=\{0\}, U=\mathbb{R}^{m}, H=\mathbb{R}^{s}, m, s \in \mathbb{N}$, and a minimum dwell time $\theta_{m}>0$, such that $\forall \tau \in \mathcal{T}, \forall I_{j} \in \tau$, $t_{j}^{\prime}-t_{j} \geq \theta_{m}$.

Definition 12: [6] $\mathbb{S}$ is observable if $\exists \varphi: \mathcal{C}\left(\mathbb{R}_{0}^{+} \times \mathbb{N}, \mathbb{R}^{s}\right)$ $\times \mathcal{C}(\mathbb{N}, P) \times \mathcal{C}^{0}\left(\mathbb{R}_{0}^{+}, U\right) \rightarrow \Xi, j^{\prime} \geq 0, \theta \in\left(0, \theta_{m}\right)$ such that $\forall \xi_{0} \in \Xi, \forall \tau \in \mathcal{T}, \forall v \in \mathcal{C}(\mathbb{N}, V)$, $\exists \chi=\left(\xi_{0}, \tau, u, d, \xi, y\right)$ such that $\varphi\left(\left.y\right|_{\left[\left(t_{0}, 0\right),\left(t, j^{\prime}\right)\right]},\left.u\right|_{\left[t_{0}, t\right)}\right)=$ $\xi(t, j), \forall t \in\left(t_{j}+\theta, t_{j}^{\prime}\right], \forall j=j^{\prime}, j^{\prime}+1, \ldots, \operatorname{card}(\tau)-1$, where $\left.y\right|_{\left[\left(t_{0}, 0\right),\left(t, j^{\prime}\right)\right]}:=y(t, j)$, if $j \leq j^{\prime},\left.y\right|_{\left[\left(t_{0}, 0\right),\left(t, j^{\prime}\right)\right]}:=$ 0 , otherwise. $\mathbb{S}$ is unobservable if it is not observable. 

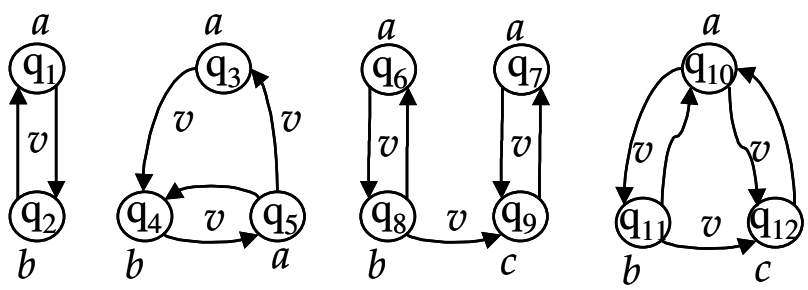

Fig. 1. DESs $\mathbb{D}_{1}, \mathbb{D}_{2}, \mathbb{D}_{3}$ and $\mathbb{D}_{4}$.

Theorem 18: [6] $\mathbb{S}$ is observable iff $\forall q_{i} \in Q, \Sigma\left(q_{i}\right)$ is observable and $\forall q_{i}, q_{j} \in Q, q_{i} \neq q_{j} \exists k \in \mathbb{N} \cup\{0\}$ : $C\left(q_{i}\right) A\left(q_{i}\right)^{k} B\left(q_{i}\right) \neq C\left(q_{j}\right) A\left(q_{j}\right)^{k} B\left(q_{j}\right)$ or $\gamma\left(q_{i}\right) \neq \gamma\left(q_{j}\right)$.

The following preliminary result holds.

Proposition 19: If $\mathbb{S}$ is observable then $\mathbb{S} \in \min (\mathbb{S})$.

Unobservable SLSs may give rise to reduced observable or unobservable SLSs. Therefore we introduce the following

Definition 13: $\mathbb{S}$ is nonproperly unobservable if it is unobservable and $\mathbb{S}^{\mathcal{R}}$ is observable, for some $\mathcal{R}$; properly unobservable if it is unobservable and $\mathbb{S}^{\mathcal{R}}$ is unobservable for any $\mathcal{R}$.

Some examples may be useful to clarify those notions.

Example 1: Consider $\mathbb{S}_{2}$ whose DES $\mathbb{D}_{\mathbb{S}_{2}}=\mathbb{D}_{2}$ is depicted in Figure 1 and where $\Sigma_{2}\left(q_{3}\right)=\Sigma_{2}\left(q_{4}\right)=$ $\Sigma_{2}\left(q_{5}\right)$ and observable and $M_{2}(e)=I, \forall e \in E_{2} . \mathbb{S}_{2}$ is unobservable and any $\mathbb{S}_{2}^{\prime} \in \min \left(\mathbb{S}_{2}\right)$, whose DES is algebraically equivalent to $\mathbb{D}_{1}$, is observable. Then $\mathbb{S}_{2}$ is nonproperly unobservable.

Example 2: Consider $\mathbb{S}_{3}$ whose DES $\mathbb{D}_{\mathbb{S}_{3}}=\mathbb{D}_{3}$ is depicted in Figure 1 and where $\Sigma_{3}\left(q_{6}\right)=\Sigma_{3}\left(q_{7}\right)=$ $\Sigma_{3}\left(q_{8}\right)=\Sigma_{3}\left(q_{9}\right)$ and $M_{3}(e)=I, \forall e \in E_{3}$. Suppose that $\Sigma_{3}\left(q_{i}\right)$ are observable $\forall q_{i}, i=6, \ldots, 9 . \mathbb{S}_{3}$ is unobservable and $\mathbb{S}_{3} \in \min \left(\mathbb{S}_{3}\right)$. Therefore $\mathbb{S}_{3}$ is properly unobservable.

Nonproper and proper unobservability can be characterized as follows.

Proposition 20: $\mathbb{S}$ is nonproperly (resp. properly) unobservable iff $\mathbb{S}$ is unobservable and any $\mathbb{S}^{\prime} \in \min (\mathbb{S})$ is observable (resp. unobservable).

Conditions of Propositions 20 may be checked easily in view of Corollary 14 and procedures of Section III. Thanks to the discussion above, reduction via hybrid bisimulation is a good tool to extract the observable SLS of any nonproperly unobservable SLS but not the right one for doing the same for properly unobservable SLSs: the key idea in this last case is to use abstraction of SLSs.

Proposition 21: For any properly unobservable $\mathbb{S}$, there exists an abstraction $\mathbb{S}^{\mathcal{A}}$ of $\mathbb{S}$ that is observable.

Example 3: Consider $\mathbb{S}_{3}$ of Example 2. Define $\mathbb{S}_{4}$, whose DES $\mathbb{D}_{\mathbb{S}_{4}}=\mathbb{D}_{4}$ is depicted in Figure 1 and where $\Sigma_{4}\left(q_{10}\right)=\Sigma_{4}\left(q_{11}\right)=\Sigma_{4}\left(q_{12}\right):=\Sigma_{3}\left(q_{6}\right)$ and $M_{4}(e)=I$, $\forall e \in E_{4}$. $\mathbb{S}_{4}$ is observable and is an abstraction of $\mathbb{S}_{3}$.

\section{CONCLUSION}

We studied bisimulation for switching linear systems. We compared equivalence by bisimulation, algebraic, statespace and I/O equivalences. An algorithm converging to the maximal hybrid bisimulation was presented. Reduction by bisimulation and abstraction by simulation were addressed and connections to observability were provided. Future work will concentrate on extensions of these results to more general hybrid systems.

Acknowledgement The first author wishes to thank Agung Julius and Rom Langerak, University of Twente, and Mihaly Petreczky, CWI, for useful discussions on bisimulations of hybrid systems.

\section{REFERENCES}

[1] Alur, R., Henzinger, T.A., Lafferriere, G., Pappas, G.J., Discrete abstractions of hybrid systems, Proceedings of the IEEE, vol. 88, pp. 971-984, 2000.

[2] Broucke, M., Regularity of solutions and homotopic equivalence for hybrid systems. Proceedings of the 37st IEEE Conference on Decision and Control, Tampa, Florida, USA, December 1998, pp. 4283-4288.

[3] Callier, F.M., Desoer, C.A.. Linear System Theory, Springer Verlag, New York, 1991.

[4] Clarke, E.M., Grumberg, O., Peled, D.A., Model Checking, The MIT Press, Cambridge, Massachusetts, 2002.

[5] De Santis, E., Di Benedetto, M.D., Berardi, L., Computation of maximal safe sets for switching linear systems, IEEE Trans. on Automatic Control, vol. 49, no. 2, February 2004. pp. 184-195.

[6] De Santis, E., Di Benedetto, M.D., Pola, G., On Observability and Detectability of continuous-time Switching Linear Systems. Proceedings of the 42st IEEE Conference on Decision and Control, Maui, Hawaii, USA, December 2003, pp. 5777-5782.

[7] Hermanns, H., Interactive Markov Chain, Lecture Notes in Computer Science 2428, Springer, 2002.

[8] Hopcroft, J.E., Ullman, J.D., Introduction to Automata Theory, Languages, and Computation, Addison Wesley Publishing Co., Reading Massachusetts, 1979.

[9] Kelley, J. L., Namioka, I., Linear Topological Spaces, Springer Verlag, 1963.

[10] Lygeros, J., Tomlin, C., Sastry, S., Controllers for reachability specifications for hybrid systems, Automatica, Special Issue on Hybrid Systems, vol. 35, 1999.

[11] Pappas, G.J., Bisimilar linear systems. Automatica, 39 (12), pp. 20352047, 2003.

[12] Pappas, G.J., Bisimilar control affine systems, Systems \& Control Letters, 52, 49-58, 2004.

[13] Pola, G., Van der Schaft, A.J., Di Benedetto, M.D., Equivalence of Switching Linear Systems by Bisimulation, Department of Electrical Engineering, University of L'Aquila, Italy, Research Report, September 2004, http://www.diel.univaq.it/tr/web/web_search_tr.php. Submitted for publication.

[14] Tabuada, P., Pappas, G.J., Lima, P., Composing abstractions of hybrid systems, in Hybrid Systems: Computation and Control, eds. C. Tomlin, M.R. Greenstreet, Lecture Notes in Computer Science, pp. 436-450, Springer, 2002.

[15] Van der Schaft, A. J., Equivalence of dynamical systems by bisimulation, Technical Report Department of Applied Mathematics, University of Twente, IEEE Trans. on Automatic Control, December 2004, to appear.

[16] Van der Schaft, A. J., Equivalence of hybrid dynamical systems, Proc. of Mathematical Theory of Networks and Systems (MTNS 04), Leuven (Belgium), July 5-9, 2004. 